2nd Galileo-XuGuangqi Meeting

International Journal of Modern Physics: Conference Series

Vol. 12 (2012) 390-399

(C) World Scientific Publishing Company

DOI: $10.1142 / \mathrm{S} 2010194512006605$

\title{
PRE INFLATION MATTER ERA AND CMB ANOMALY
}

\author{
FABIO SCARDIGLI $^{a}$, CHRISTINE GRUBER $^{a}$ and PISIN CHEN ${ }^{a b}$ \\ ${ }^{a}$ Leung Center for Cosmology and Particle Astrophysics (LeCosPA), \\ National Taiwan University, Taipei 106, Taiwan. \\ ${ }^{b}$ Kavli Institute for Particle Astrophysics and Cosmology, \\ SLAC National Accelerator Laboratory, Stanford University, Menlo Park, CA 94025, U.S.A. \\ fabio@phys.ntu.edu.tw, chrisy_gruber@gmx.net, chen@slac.stanford.edu
}

\begin{abstract}
We consider the production of primordial micro black holes (MBH) remnants in the early universe. These objects induce the universe to be in a matter-dominated era before the onset of inflation. Effects of such an epoch on the CMB power spectrum are discussed and computed both analytically and numerically. By comparison with the latest observational data from the WMAP collaboration, we find that our model appears to explain the quadrupole anomaly of the CMB power spectrum.
\end{abstract}

Keywords: black hole remnants; pre inflation era; CMB anomaly.

\section{Introduction}

Inflation is without doubt the best model to explain the observed spatially flat and homogeneous Universe. Nevertheless, despite the great successes of the standard $\Lambda \mathrm{CDM}$ model in explaining almost all the data on CMB anisotropy as most recently measured by WMAP observations, the suppression of the $l=2$ quadrupole mode still remains a puzzle in the framework of the standard $\Lambda$ CDM model (for a review on this subject, see e.g. Ref. 1). In the present paper we propose a pre-inflationary scenario that is based on a generic micro black hole $(\mathrm{MBH})$ production and a minimal set of first principles, namely the generalized uncertainty principle (GUP) and the holographic principle (HP), and it appears to give rise to the suppression of the CMB quadrupole self-consistently without the need of arbitrary inputs. Specifically, we consider the possibility of production of micro black holes in the early pre-inflationary Universe, due to quantum fluctuations of the metric field ${ }^{4,5}$, as the seeds for the suppression of the inflaton fluctuations. There are two salient features of this $\mathrm{MBH}$ nucleation. One is that the production rate per unit volume of space and time is very high at the Planck temperature. To prevent unphysical over-production of $\mathrm{MBH}$, we invoke the holographic principle (HP) to constrain the initial condition of $\mathrm{MBH}$ production. The other is that the rate of such $\mathrm{MBH}$ production is exponentially suppressed when the temperature of the universe is sufficiently below the Planck temperature. Inflation is in general assumed to start when the temperature of the universe reaches the scale of the GUT energy, about $10^{15}-10^{16} \mathrm{GeV}$. 
Therefore one expects that the MBH production activity would cease long before the onset of the inflation, and the $\mathrm{MBH}$ would have been totally evaporated and the universe would turn into radiation era before the inflation begins. However, when the Generalized Uncertainty Principle (GUP) is taken into consideration, the complete decay of the nucleated $\mathrm{MBH}$ into radiation is prevented, and we have massive, but inert black hole remnants ${ }^{6}$ populating the pre-inflationary phase of the Universe. Furthermore, the nucleation of $\mathrm{MBH}$ is so efficient and fast that the Universe is put into a matter dominated era within a few Planck times, just about $10^{3} t_{p}$ after the Big Bang (i.e., well before inflation) and there the Universe stays until the onset of inflation. Such a pre-inflation matter-dominated universe then suppresses the initial inflaton fluctuations at the onset of the inflation. In order to isolate the cause of the CMB quadrupole anomaly, we further examine one variation of this model, one without the GUP, where the black holes decay into radiation completely. In all two cases the primordial power spectra have been fed to the CMBFAST code in order to obtain the CMB power spectra, and then compared with each other and tested against the last WMAP observational data. The pre-inflation matter model seems to be the only one, among those studied, which is able to describe the $l=2$ mode suppression, although the radiation model still presents a better fitting of the data at high $l$ values. Throughout the paper the Planck length is defined as $\ell_{p}^{2}=G \hbar / c^{3}$, the Planck energy as $\mathcal{E}_{p} \ell_{p}=\hbar c / 2$, and the Planck mass as $M_{p}=\mathcal{E}_{p} / c^{2}$.

\section{Black hole Physics with Generalized Uncertainty Principle}

As it is well known from the classical argument of the Heisenberg microscope ${ }^{7}$, the size $\delta x$ of the smallest detail of an object, theoretically detectable with a beam of photons of energy $E$, is roughly given by $\delta x \simeq \hbar c /(2 E)$. The research on viable generalizations of the Heisenberg uncertainty principle traces back to many decades (see Refs. 8, 9, and for more recent approaches Ref. 10, 11, 12). The results, both in string theory and in gedanken experiments with micro black holes, are summarized by the formula

$$
\delta x \gtrsim \frac{\hbar c}{2 E}+\beta \ell_{p} \frac{E}{\mathcal{E}_{p}},
$$

where $\beta$ is the deformation parameter, generally believed to be of $O(1)$. Following loosely the arguments of Refs. 14, 6, 17, 15, 16, 13, defining the Planck temperature $T_{p}$ so that $\mathcal{E}_{p}=k_{B} T_{p} / 2$, and measuring all temperatures in Planck units as $\Theta=$ $T / T_{p}$, we can infer from (1) the relation between the mass $m$ and the temperature $\Theta$ of a black hole, as having the form

$$
2 m=\frac{1}{2 \pi \Theta}+\zeta 2 \pi \Theta
$$

where we have defined the deformation parameter $\zeta=\beta / \pi^{2}$. In the semiclassical limit both $\beta$ and $\zeta$ tend to zero and Eq. (2) boils down to $m=1 /(4 \pi \Theta)$, which is the dimensionless version of Hawking's formula. The standard Hawking formula predicts a complete evaporation of a black hole, from an initial mass $M$ down 
to zero mass. However, the GUP formulation immediately leads to a minimum mass and a maximum temperature for the evaporating black hole. Precisely we have $\Theta_{\max }=1 /(2 \pi \sqrt{\zeta}), m_{\min }=\sqrt{\zeta}$. Note that, as expected, $\Theta_{\max } \rightarrow \infty$ and $m_{\text {min }} \rightarrow 0$ in the Hawking limit $\beta \rightarrow 0$. Therefore the use of the GUP eliminates the problem of an infinite temperature at the end of the evaporation process, which is clearly unphysical, and leads directly to the prediction of the existence of black hole remnants (see Refs.6, 15, 17, 18, 19).

\section{Pre-inflation matter era and inflationary solutions}

We consider the standard RW flat metric (with Weinberg conventions but $c \neq 1$ ) $d s^{2}=-c^{2} d t^{2}+a^{2}(t)\left(d r^{2}+r^{2} d \Omega^{2}\right)$, where $d \Omega^{2}=d \theta^{2}+\sin ^{2} \theta d \phi^{2}$. Following Ref. 3, the (00) component of the Einstein equation in this case reads

$$
\left(\frac{\dot{a}}{a}\right)^{2}=\kappa\left(\frac{A}{a^{3}}+\frac{B}{a^{4}}+C\right) .
$$

where we consider a matter content $A$, a radiation content $B$, and a constant vacuum energy, $C$, namely a cosmological constant. In this way, we shall be able to generate inflationary exponential solutions. The constant $C$ in the Friedmann equation is mimicking the potential for the inflaton field. We can read off the matter era dominance condition as $B /(A a) \ll 1$ (or $B=0$ ). Under this condition Eq.(3) is easily separable, and the solution ${ }^{20}$ obeying the boundary condition $a(0)=0$ for $t=0$ is

$$
a(t)=\left(\frac{A}{C}\right)^{1 / 3}\left[\sinh \left(\frac{3}{2} \sqrt{\kappa C} t\right)\right]^{2 / 3},
$$

which, for vanishing $C$, or small $t$, results in the solution $a(t) \sim t^{2 / 3}$.

In case of radiation dominated pre-inflation era, i.e. no matter present $(A=0)$, the solution obeying the boundary condition $a(0)=0$ for $t=0$ is

$$
a(t)=\left(\frac{B}{C}\right)^{1 / 4}[\sinh (2 \sqrt{\kappa C} t)]^{1 / 2},
$$

which for small $t$ or vanishing $C$ behaves as $a(t) \sim t^{1 / 2}$.

\section{Black Hole Nucleation}

In 1982 Gross, Perry and Yaffe ${ }^{4}$, and two years later, Kapusta ${ }^{5}$, investigated the stability of flat spacetime and the arising of gravitational instabilities, which might lead to singularities and to spontaneous formation of black holes. They found a nucleation rate for black holes as

$$
\Gamma_{N}(\Theta)=\frac{1}{15 \cdot 8 \pi^{2}} \cdot \Theta^{-167 / 45} \mathrm{e}^{-1 / 16 \pi \Theta^{2}},
$$

where $\Theta$ is the temperature of the universe (the thermal bath), expressed in Planck units, and at the same time the temperature of the nucleated black holes, connected to their mass $m$ by $\Theta=\frac{1}{4 \pi m}$. At a given temperature $\Theta$, all the black holes created 
according to this nucleation rate will have mass $m$. At very early times, when $\Theta>\Theta_{*} \simeq 1 /(4 \pi)$, the nucleation probability is very high, but does not lead to black hole formation, since it is forbidden by the GUP to create smaller than one Planck mass black holes $\left(m_{\min } \simeq \sqrt{\zeta} M_{p}\right.$ where $\left.\zeta \sim 1\right)$. So at least for this very early time, the universe is simply a chaotic hot sphere, filled with primordial radiation (see Refs.2, 3). Considering an adiabatically expanding universe, we can write $T(\tau) a(\tau)=T_{p} a\left(t_{p}\right)$, and since during the radiation era the scale factor evolves like $a_{r}(\tau) \simeq a\left(t_{p}\right) \tau^{1 / 2}$, and we choose $a\left(t_{p}\right)=1$, we have $\Theta_{r}=1 / \tau^{1 / 2}$ for a radiation dominated universe. In the matter-dominated era, the temperature evolution is analogously given by $\Theta_{m}=1 / \tau^{2 / 3}$. In radiation era we can write the nucleation rate as a function of time as $\Gamma_{N, r}(\tau)=\left(15 \cdot 8 \pi^{2}\right)^{-1} \cdot \tau^{167 / 90} \mathrm{e}^{-\tau / 16 \pi}$. The parameters in the Friedmann equation containing matter and radiation energy densities are defined as $A=\rho_{m}\left(\tau_{c}\right) \cdot a^{3}\left(\tau_{c}\right), \quad B=\rho_{r}\left(\tau_{p}\right) \cdot a^{4}\left(\tau_{p}\right)$, and their dimension has to be energy per volume, as the scale factor is a dimensionless quantity. At the Planck time, the universe is in a radiation-dominated stage, and it is reasonable to assume that $\rho_{r}\left(\tau_{p}\right)=\rho_{p}$. Assuming $a\left(\tau_{p}\right)=1$, the radiation parameter can be fixed as $B=1$, expressed in Planck units. For the matter parameter $A$, we have to choose a time $\tau_{c}$ when black holes are starting to be nucleated, and calculate $\rho_{m}\left(\tau_{c}\right)$. This time and the parameter $A$ will be derived via numerical simulations.

\subsection{A Cutoff from the Holographic Principle}

The holographic principle ${ }^{21}$ places a limit on the information content, or entropy content, in a certain region of space-time. Quantitatively it states that (in units where $\left.k_{B}=\ell_{p}=1\right) S[L(B)] \leqslant A(B) / 4$, where $L(B)$ is a so-called light sheet, which defines a certain region of space-time $B$, and $A(B)$ is the codimension 1 boundary of that region. This can be used to define a cutoff for the nucleation rate of micro black holes. We demand that at no time point in the evolution of the universe the entropy of the black holes can exceed the total entropy that Hubble sphere can maximally hold. Then we try to find a time point $\tau_{c}$, from which this condition is fulfilled. If this condition is violated in the course of black hole production, then it is simply not allowed to create black holes. We require that at all times $S_{b h}(\tau) \leqslant S_{H S}(\tau)$. The universe has started off in a radiation-dominated era, so the nucleation rate as a function of time is computed using $\Theta_{r}=\tau^{-1 / 2}$. The cutoff time turns out to be $\tau_{c}=993$. With this assumption the estimated number of primordial black holes is given by $N\left(\tau_{\text {inf }}\right) \sim 10^{4}$. The masses of the black holes nucleated between $\tau_{c}=993$ and $\tau=998$ are on average $m\left(\tau_{c}\right) \sim 2.5$.

\subsection{Fixing the Friedmann Equation}

In this subsection we determine the numerical size of the parameters in the Friedmann equation of our model. We have already settled $B=1$. For the matter component, we know that about $10^{4}$ black holes are nucleated during a short period around $\tau \sim 10^{3}$ in the pre-inflation era. $A$ is given in Section 4 . The matter density can be simply estimated by $\rho_{m}\left(\tau_{c}\right) \sim\left[10^{4}\right.$ black holes $] / R_{H}^{3}\left(\tau_{c}\right) \sim\left(10^{4} \sqrt{\zeta} \epsilon_{p}\right) /\left(10^{9} V_{p}\right)$, 
where $m_{\min }=\sqrt{\zeta}$ is the minimum mass predicted by the GUP. The scale factor is chosen as $a\left(t_{p}\right)=1$, and then evolves in radiation-dominance until $\tau_{c} \sim 10^{3}$; thus $a\left(\tau_{c}\right) \sim \tau_{c}^{1 / 2} \sim 10^{3 / 2}$. Putting these together, the final result for the matter component is $A \sim 10^{-1 / 2} \sqrt{\zeta} \epsilon_{p} / V_{p}$. As stated before (see Sections 2), we consider $\zeta \sim 1$. With this number the matter-dominance ratio spans the values $B /(A \cdot a(\tau)) \sim 10^{-1}-10^{-3} \ll 1$, which confirms that the Universe is in matter era from $\tau_{c}$ to the onset of inflation.

\section{Scalar field fluctuations - The primordial power spectrum}

In this section we study the quantum fluctuations of a field living in a universe whose background evolves with a given scale factor $a(t)$. We choose the same convention on the metric as in Ref.3, namely $d s^{2}=g_{\mu \nu} d y^{\mu} d y^{\nu}=d t^{2}-a(t)^{2} d \vec{y}^{2}$. Here we set $c=1$, and we choose a flat metric (for simplicity, and since we deal with an almost flat universe). We consider a zero mass scalar field $\Phi(t, \vec{y})$ and we perturb the field around its classical expectation value, $\Phi(t, \vec{y})=\Phi_{0}(t, \vec{y})+\varphi(t, \vec{y})$. The equation of motion for the scalar field perturbation then reads $\square \varphi(t, \vec{y})=0$. In the applications, the $a(t)$ of Eq.(4), or of Eq.(5), will be used in the metric $g_{\mu \nu}$. The equation of motion for the Fourier transformed field $\phi_{k}(t)$ reads

$$
\ddot{\phi}_{k}(t)+3 \frac{\dot{a}}{a} \dot{\phi}_{k}(t)+\left(\frac{k^{2}}{a^{2}}\right) \phi_{k}(t)=0 .
$$

A possible strategy to solve Eq.(7) is to change the independent variable from $t$ to $a$. Then the equation for $\phi_{k}(a)$ reads

$$
\dot{a}^{2} \phi_{k}^{\prime \prime}+\left(\ddot{a}+3 \frac{\dot{a}^{2}}{a}\right) \phi_{k}^{\prime}+\left(\frac{k^{2}}{a^{2}}\right) \phi_{k}=0,
$$

where of course $\dot{a}$ and $\ddot{a}$ have to be expressed as functions of $a$.

\subsection{The re-entering $k$-modes}

The k-modes which left the horizon at, or shortly after, the onset of inflation are those that are just now re-entering our Hubble radius, and they represent the largest modes of fluctuations currently observable, having a size comparable with that of the visible universe. The modes leaving the horizon just at, or shortly after, the beginning of inflation, correspond to the largest scales observable today, and they could bring imprints of a possible pre inflation era. A pre-inflationary era affecting these modes could thus explain the anomaly of the quadrupole moment of the CMB today. Simple geometric considerations help us to find a relation between $k$ and the scale factor, $k \simeq a H$, as the horizon crossing condition. Besides, reminding that $H=\dot{a} / a$, we have for a pre-inflation radiation era

$$
k=a \sqrt{\kappa}\left(\frac{B}{a^{4}}+C\right)^{1 / 2}
$$


while, for a pre-inflation matter era,

$$
k=a \sqrt{\kappa}\left(\frac{A}{a^{3}}+C\right)^{1 / 2} .
$$

\subsection{Scalar Field equations}

In this subsection we specialize Eq.(8) to particular $a(t)$ solutions. For the pre inflation matter era case, using Eq.(4) to compute $\dot{a}, \ddot{a}$, we obtain

$$
\phi_{k}^{\prime \prime}+\frac{1}{a}\left(\frac{4 C a^{3}+\frac{5}{2} A}{C a^{3}+A}\right) \phi_{k}^{\prime}+\left(\frac{k^{2}}{\kappa a\left(C a^{3}+A\right)}\right) \phi_{k}=0 .
$$

In the case of pre inflation radiation era we have, using Eq. (5),

$$
\phi_{k}^{\prime \prime}+\frac{2}{a}\left(\frac{C a^{4}}{C a^{4}+B}+1\right) \phi_{k}^{\prime}+\left(\frac{k^{2}}{\kappa\left(C a^{4}+B\right)}\right) \phi_{k}=0 .
$$

The general plan of our work is to solve equations (11), (12), regarding $k$ as a parameter, to obtain $\phi(a, k)$. Once the solution $\phi(a, k)$ is available, we can compute the power spectrum $P(k)$ of the quantum fluctuations of the field $\Phi$. Since the power spectrum is usually given as a function of $k$ only, will be necessary to express $\phi$ as a function of $k$ only, and this can be done through the relations (9), (10). The final scope is to obtain the function $P(k)=k^{3}|\phi(a(k), k)|^{2}$, which represents the primordial power spectrum of the fluctuations (perturbations) of the field $\Phi$. With $P(k)$ then we feed the CMBFAST code to generate the CMB anisotropy power spectra. Luckily enough, it turns out that analytical solutions of the second order approximations of equations (11),(12) can be obtained by means of the WKB method, and these solutions are strongly corroborated by numerical insights. Of course, when solving a differential equation, we need boundary conditions in order to fix the solution explicitly. Since we are almost sure that inflation happened, while we know little about a possible pre-inflation era, we choose to put our boundary conditions in the full inflationary era. This means that, for large $a$, or equivalently, for large $k$ (see equations (9), (10)), well after the beginning of inflation, the field $\phi$ must generate an almost scale invariant, i.e. flat, primordial power spectrum $P(k)$. The newest analysis of the WMAP data ${ }^{23}$ indicate a form like $P(k) \sim k^{n_{s}-1}$ with $n_{s}=0.963 \pm 0.012(68 \% C L)$. Therefore, for large $k$ the field $\phi$ must behave as $|\phi(a(k), k)| \sim k^{\frac{1}{2}\left(n_{s}-1\right)-\frac{3}{2}}$ This condition will allow us to fix properly the arbitrary constants in our solutions.

\subsection{WKB solutions}

We develop the coefficients of Eqs.(11) (12) to the second order in $a$ for $a \rightarrow \infty$. Then we apply the WKB method. In this way the WKB solution of Eq.(11) explicitly reads

$$
\phi_{k}(a)=\frac{2 \sqrt[4]{\kappa C^{2}}\left[c_{+}(k) e^{i G(a)}+c_{-}(k) e^{-i G(a)}\right] e^{-i \pi / 4}}{\left[32 \kappa C^{2} a^{6}-16 k^{2} C a^{4}+9 \kappa A^{2}\right]^{1 / 4} \cdot \exp \left[A /\left(4 C a^{3}\right)\right]}
$$


We can always find agreement with the boundary condition $|\phi| \sim k^{-3 / 2+\left(n_{s}-1\right) / 2}$ (for large $k$ ), by defining the arbitrary constants $c_{ \pm}(k)$ accordingly. In fact for large $k$, we can choose $c_{ \pm}(k)$ in a way that $\left[c_{+}(k) e^{i G(a(k))}+c_{-}(k) e^{-i G(a(k))}\right] \sim k^{\left(n_{s}-1\right) / 2}$ so that the boundary condition is fulfilled. We can also consider relation (10) to the second order of approximation, which reads $a(k) \simeq \frac{k}{\sqrt{\kappa C}}-\frac{A \kappa}{2 k^{2}}$. Once this new expression of $a(k)$ is substituted in (13), it is instructive to plot the function $P=k^{3}|\phi(k)|^{2}$. An analogous procedure can be applied to the pre-inflation radiation era, starting from Eq.(12). Finally, in Fig.1, we compare the plots for the power spectra, matter and radiation cases, in the same diagram. We see that for both matter and radiation eras there is an exponential suppression of the low $k$-modes. The matter diagram presents an interesting cusp, just before dropping down, that is absent in the radiation diagram.

\subsection{Numerical computation of the primordial power spectrum}

Matter era - In one of the previous sections we determined the parameter of the matter contribution in the Friedmann equation, $A$, to be $A=10^{-1 / 2} \epsilon_{p} / V_{p}$. After a radiation-dominance era and a subsequent period of matter-dominance before inflation, we can, with the assumption of $a\left(t_{p}\right)=1$, compute the scale factor at the onset of inflation $a_{i n f}=10^{7 / 2}$. When inflation starts, the two competing factors of matter and inflation in the Friedmann equation, namely $A / a^{3}$ and $C$, must be of the same order of magnitude. This condition allows us to fix the coefficient $C$ as $C=A /\left(2 a_{\text {inf }}^{3}\right)=0.5 \cdot 10^{-11} \epsilon_{p} / V_{p}$. The boundary conditions for the numerical solution have been obtained in section 5.2. The numerical solution of Eq.(11), as a function of $k$, is however only a collection of data points. In order to be of any use for the CMBFAST code, it has to be given as an analytical function, which is obtained by fitting the data points with an opportune mathematical function.

Radiation era with totally evaporating black holes - Here we deal with the situation in which black holes are nucleated but then disappear again completely

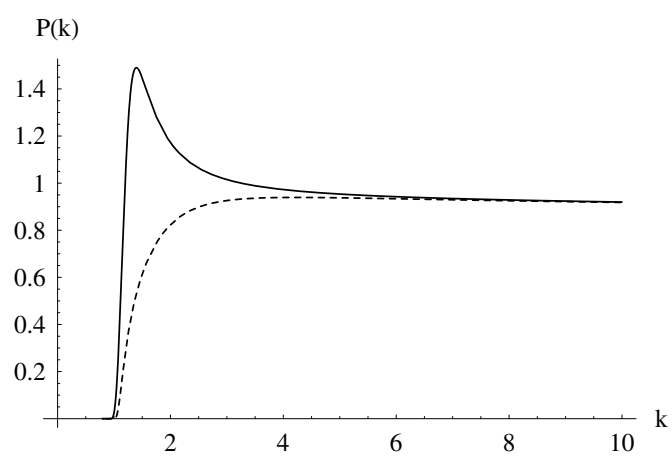

Fig. 1. Primordial power spectra $P(k)$ versus $k$, for pre-inflation matter (full/upper line) and radiation (dashed/lower line) eras. 


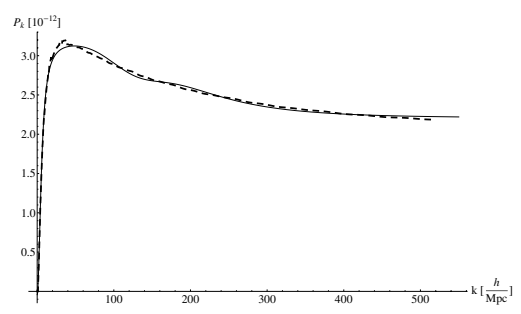

Fig. 2. The numerical solution for the primordial power spectrum (dashed line) and its fitting function (full line) in the pre-inflation matter era scenario.

into radiation. We can determine the parameters $B$ and $C$ in this case. When $\tau$ spans the interval between the end of black hole evaporation, $\tau_{r} \simeq 10^{4}$, and the onset of inflation, $\tau_{\text {inf }} \simeq 10^{8}$, then the scale factor evolves in full radiation era as $a(\tau)=a\left(t_{p}\right)\left(\tau_{r} / \tau_{c}\right)^{1 / 6} \tau^{1 / 2}$. Reminding $a\left(t_{p}\right)=1$ and $\tau_{c} \simeq 10^{3}$ we get a scale factor at the onset of inflation of $a\left(\tau_{\text {inf }}\right)=10^{25 / 6} \sim 10^{4.16}$. The radiation content of the Universe is fixed at $\tau_{r}$, the end of the black hole evaporation era. Since by then $R_{H}=10^{4} \ell_{p}$, thus we have $B=\rho_{r}\left(\tau_{r}\right) \cdot a^{4}\left(\tau_{r}\right)=10^{2 / 3} \sqrt{\zeta} \epsilon_{p} / V_{p}$. Here, as before, we choose again $\zeta \sim 1$. Hence, the inflationary parameter $C$ can be fixed by requiring that it is of the same order as the radiation parameter $B$ at the onset of inflation $C \simeq B / a_{\text {inf }}^{4} \sim 10^{-16}$. Thus we can now numerically solve the equation for a scenario without GUP in the same way as before.

\section{The CMB power spectrum}

The previously calculated primordial power spectra, in the cases with GUP, and without GUP, have been fed into the CMBFAST code $^{22}$ to obtain the CMB temperature anisotropy spectrum that we can measure today. We compare our results to the WMAP seven year data, and to the result for the CMB spectrum obtained from the standard inflationary theory, without any pre-inflation era. The standard inflation (SI) model is a very good fit to the data, considering all the different features it has to explain. Only the point for the mode with $l=2$ in the CMB spectrum is very low in comparison with the theoretical curve. To produce the CMB power spectrum corresponding to the SI model, we assumed a scalar spectral index of $n_{s}=0.963$ and a running of the index $\alpha_{n_{s}}=-0.022$, i.e. the most recent result of the WMAP observations ${ }^{23}$. The number $N_{o}$ of e-folds of inflation can in principle be varied in computing the CMB power spectrum. The observational lower bound on $N_{o}$ stated in Ref. 24 is $N_{o}=54 \pm 7$. The numerical results for the CMB power spectrum are the following. For a matter-dominated era before inflation, the CMB power spectrum as obtained by CMBFAST can be seen in Fig.3. For the case when the GUP is turned off, the CMB power spectrum can be seen in Fig.4. 

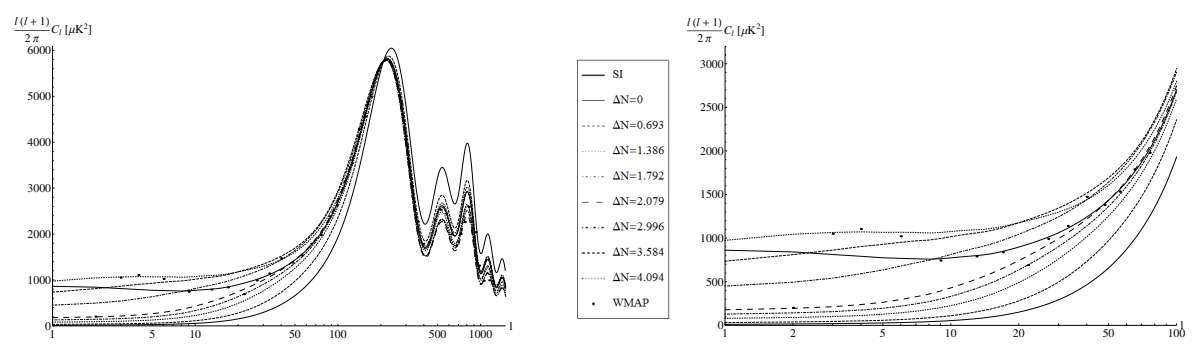

Fig. 3. The CMB power spectrum for a pre-inflation matter era, for various cases of $\Delta N$. Overall view and zoom into the lower multipole region.
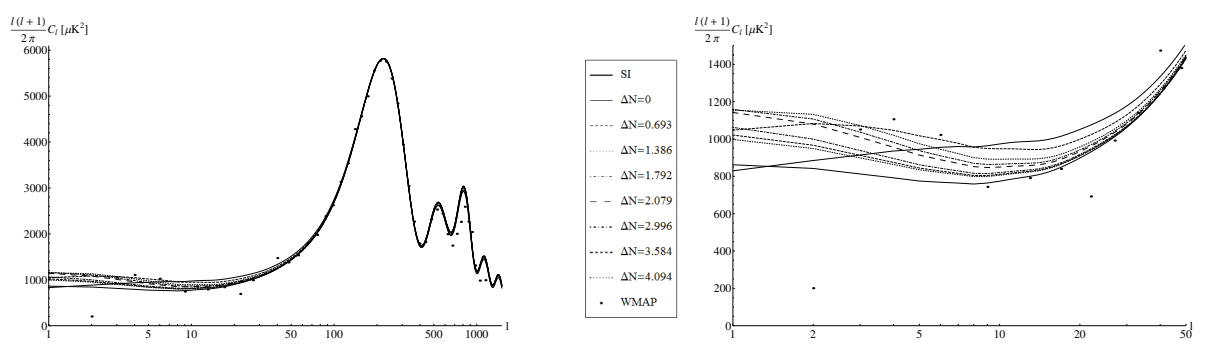

Fig. 4. The CMB power spectrum for a pre-inflation radiation era, for various cases of $\Delta N$. Overall view and zoom into the lower multipole region.

\section{Conclusions and Outlook}

In this work, we investigated the effects of an era before inflation on the CMB power spectrum measured today. We utilized the phenomenon of black hole nucleation from quantum fluctuations of the metric in very early times to argue for the existence of several thousand micro black holes in the pre-inflation era, which cause the universe to be matter-dominated from about $\tau \simeq 10^{3} t_{p}$, until the onset of inflation $(\tau \simeq$ $\left.10^{6} t_{p}\right)$. The model with matter-dominance in pre-inflation era is successful in the suppression of the $l=2$ mode, and incorporates the desired effect on the power spectrum very well (see Fig.3). It asymptotes to the standard inflationary model for higher $\Delta N$, as expected. For the case of radiation in pre-inflation era, with black holes evaporating until zero mass (no GUP), the result is rather poor - only one of the curves shows a suppression in the lower modes, all of them actually lie above the result given by a standard inflationary scenario without a pre-inflation era. The model shows a very good accordance for the high $l$ region. The lower bound on the number $N_{o}$ of e-folds is currently fixed by literature as $N_{o}=54 \pm 7$ e-folds. From Fig.3, we see that, in our model, in order to fit the WMAP data point at $l=2$, i.e. the so called quadrupole anomaly, we have to add about $\Delta N=2$ or $\Delta N=3$ e-folds to the standard number $N_{o}$, though still within the uncertainty 
shown above. We regard this as a bonus from our theory, which, for the first time to our knowledge, fixes an upper bound to the number of e-folds needed in inflation so as to fit the points of CMB power spectrum. Moreover, the necessity of a pre inflation matter era is further supported by observing that, as Fig.4 displays, if a pre inflation radiation era is assumed, then the curve of the CMB power spectrum cannot be lowered down to catch the anomalous $l=2$ point, no matter how many e-folds one adds to the standard number $N_{o}$. The pre inflation matter era model seems to be the only one, among those studied, able to capture and describe the $l=2$ mode suppression, although the radiation model still presents a better fitting of the data at high $l$ values.

\section{Acknowledgments}

The authors thank Kin W. Ng and Ron J. Adler for enlightening conversations. This research is supported by Taiwan NSC under Project No. NSC 97-2112-M-002026-MY3 and by US DoE under Contract No. DE-AC03-76SF00515.

\section{References}

1. K.T. Inoue, http://www.rist.kindai.ac.jp/no.19/inoue.pdf (2007).

2. B. A. Powell and W. H. Kinney, Phys. Rev. D, 76, (2007) 063512.

3. I-C.Wang, K.-W. Ng, Phys. Rev. D 77, (2008) 083501. [arXiv:0704.2095]

4. D.J.Gross, M.J.Perry, L.G.Yaffe, Phys.Rev.D 25, 330 (1982).

5. J.I.Kapusta, Phys.Rev.D 30, 831 (1984).

6. R.J.Adler, P.Chen, D.I.Santiago, Gen. Rel. Grav. 332101 (2001).

7. W.Heisenberg, Z.Phys. 43, 172 (1927).

8. C.N. Yang, Phys. Rev. 72, 874 (1947). F. Karolyhazy, Nuovo Cim. A 42, 390 (1966).

9. L.J. Garay, Int. J. Mod. Phys. A 10, 145 (1995).

10. M.Maggiore, Phys. Lett. B 304, 65 (1993).

11. F. Scardigli, Phys. Lett. B 452, 39 (1999).

12. R.J.Adler, D.I.Santiago, Mod.Phys.Lett. A14 (1999) 1371.

13. F.Scardigli, Glimpses on the micro black holes Planck phase, arXiv:0809.1832.

14. F.Scardigli, Nuovo Cim. B 110, 1029 (1995). F.Scardigli, arXiv:gr-qc/0607010.

15. M.Cavaglia, S.Das, R.Maartens, Class. Quant. Grav. 20, L205 (2003).

16. K.Nouicer, Class. Quantum Grav. 24, 5917 (2007).

17. M.Cavaglia', S.Das, Class. Quant. Grav. 214511 (2004) [arXiv:hep-th/0404050].

18. P.Chen, R.J.Adler, Nucl. Phys. Proc. Suppl. 124, 103 (2003) [arXiv:gr-qc/0205106].

19. R.Banerjee, S.Ghosh, Phys. Lett. B 688, 224 (2010) [arXiv:1002.2302].

20. A.Vilenkin, L.H.Ford, Phys. Rev. D 26, (1982) 1231.

21. G.'t Hooft, Dimensional reduction in quantum gravity, arXiv:gr-qc/9310026 (1993); L. Susskind, J.Math.Phys. 36, (1995) 6377; R. Bousso, Rev.Mod.Phys. 74, (2002) 825.

22. U. Seljak and M. Zaldarriaga, Astrophysical J., 469, (1996) 437.

-23. E. Komatsu, K. M. Smith, and J. Dunkley, Astrophys.J.Supp. 192 (2011) 18.

24. L. Alabidi and D. Lyth, JCAP, 8 (2006) 006. 